\title{
Persistent Primary Aldosteronism Despite latrogenic Adrenal Hemorrhage After Adrenal Vein Sampling
}

\author{
Keisuke Okamura ${ }^{\mathrm{a}, \mathrm{e}}$, Tetsu Okuda ${ }^{\mathrm{a}}$, Kazuyuki Shirai ${ }^{\mathrm{a}}$, Ichiro Abe ${ }^{\mathrm{b}}$, Kunihisa Kobayashi ${ }^{\mathrm{b}}$, \\ Tatsu Ishiic, Seiji Haraoka ${ }^{d}$, Hidenori Urata ${ }^{a}$
}

\begin{abstract}
Before surgery for primary aldosteronism (PA), localization is evaluated with adrenal vein sampling (AVS). A 56-year-old Japanese woman had a right adrenal mass, hypokalemia, and a high aldosterone/renin ratio. Stress tests confirmed the diagnosis of PA. Subsequently, preoperative AVS was performed and right adrenal hemorrhage (AH) occurred unexpectedly. Because hypertension persisted, laparoscopic right adrenalectomy was performed. Postoperatively, the blood pressure was normalized. Pathological examination revealed an adrenal cortical adenoma largely unaffected by necrosis and hemorrhage. Previous reports have also indicated that $\mathrm{AH}$ may not ameliorate PA. We discussed the clinical progress of $\mathrm{AH}$ and the measures to prevent causing $\mathrm{AH}$.
\end{abstract}

Keywords: Adrenal vein sampling; Adrenal hemorrhage; Primary aldosteronism

\section{Introduction}

Primary aldosteronism (PA) is a cause of secondary hypertension that accounts for $5-10 \%$ of hypertension [1]. Patients with PA can develop cardiovascular events unrelated to high blood pressure (BP) [2]. In addition to aldosterone-producing adenoma (APA), which can be diagnosed by CT scanning, PA can be caused by aldosterone-producing microadenoma and by idiopathic hyperaldosteronism. Patients can also have a non-functioning adenoma. Accordingly, adrenal vein sampling (AVS) is essential before surgical treatment [3-6], since the wrong adrenal gland is selected in $37.8 \%$ of patients when localization is only based on imaging modalities such as CT and magnetic resonance imaging (MRI) [7].

Manuscript submitted October 25, 2017, accepted November 7, 2017

aDepartment of Cardiovascular Diseases, Fukuoka University Chikushi Hospital, Japan

${ }^{b}$ Department of Endocrinology and Diabetes Mellitus, Fukuoka University Chikushi Hospital, Japan

'Department of Urology, Fukuoka University Chikushi Hospital, Japan ${ }^{\mathrm{d}}$ Department of Pathology, Fukuoka University Chikushi Hospital, Japan ${ }^{\mathrm{e} C}$ Corresponding Author: Keisuke Okamura, Department of Cardiovascular Diseases, Fukuoka University Chikushi Hospital, 1-1-1, Zokumyoin, Chikushino, Fukuoka 818-8502, Japan. Email: okamurakmd@cis.fukuoka-u.ac.jp
One of the important complications of AVS is adrenal hemorrhage $(\mathrm{AH})$, which is more likely to occur on the right side [8]. This is possibly because the left adrenal vein empties into the left renal vein and engagement of a catheter is relatively easy, while the right adrenal vein is smaller in diameter and runs directly to the inferior vena cava, thereby making catheter engagement more difficult [9]. Here we report the details of a patient with AH due to AVS and discuss preventive measures for this complication.

\section{Case Report}

The patient was a 56-year-old Japanese woman. Hypertension was diagnosed by a local doctor when she was around 42 years old and oral medication was started. At the age of 56 years, ultrasound identified a right adrenal mass during a routine medical check-up and CT confirmed a $1.4 \mathrm{~cm}$ adrenal mass (Fig. 1). Laboratory tests revealed hypokalemia $(3.4 \mathrm{mmol} / \mathrm{L})$. En-
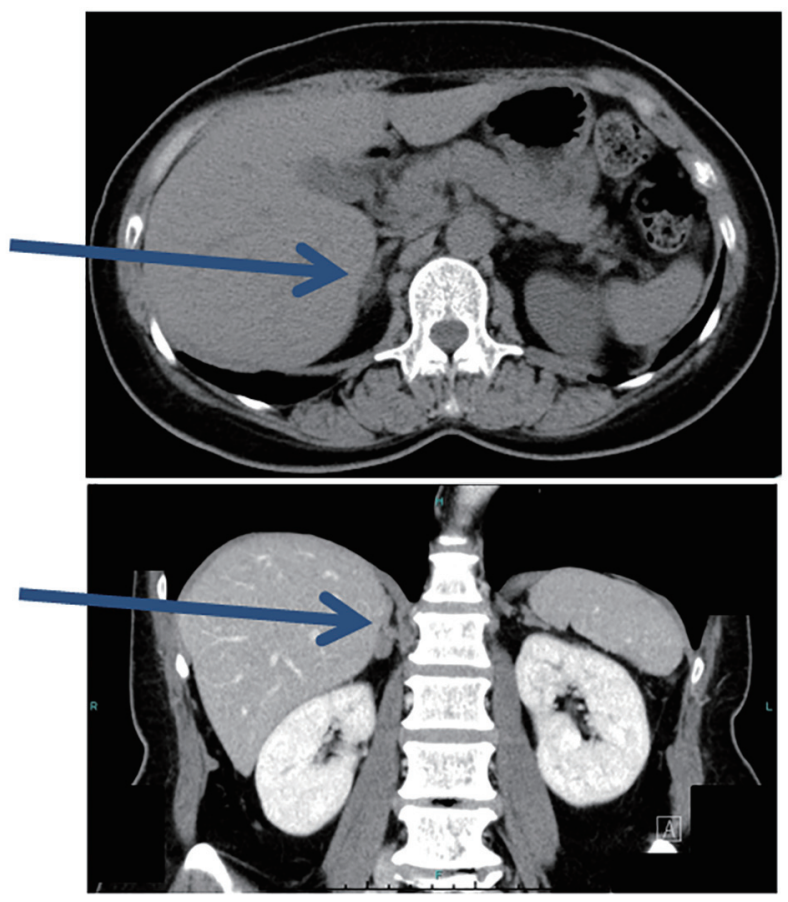

Figure 1. Plain CT prior to AVS. CT detected an adrenal mass $1.4 \mathrm{~cm}$ in diameter (arrows). 

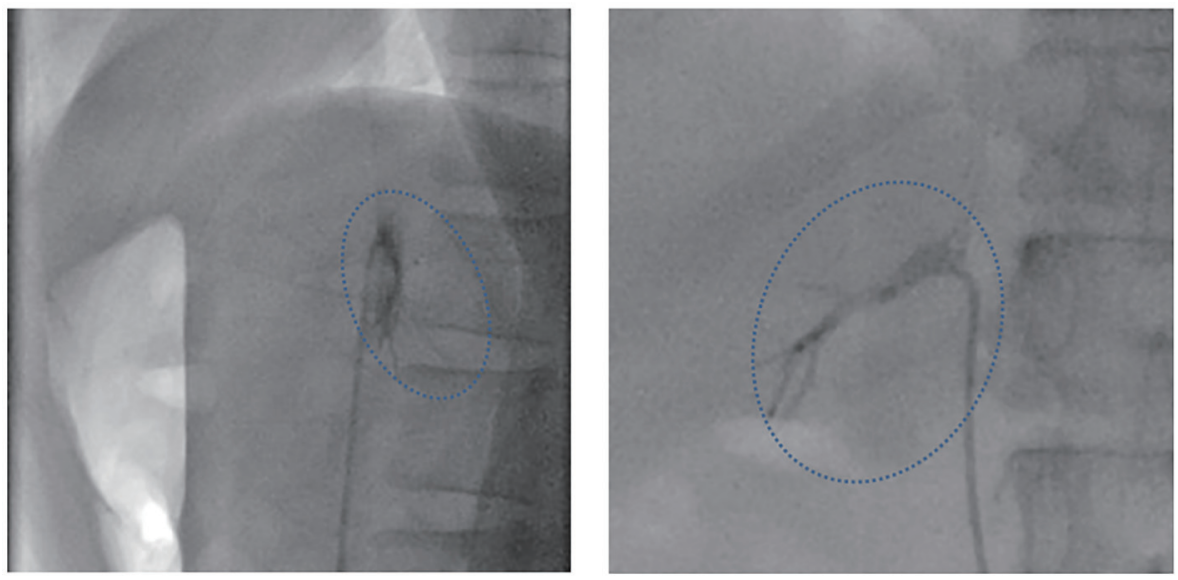

Figure 2. Fluoroscopy images immediately after AVS. Left: LAO90, CRA0; right: RAO90, CRA0. The patient complained of chest discomfort and back pain after AVS, and pooling of contrast medium was noted at that time in the entire right adrenal gland (dotted circle).

docrine studies demonstrated a high plasma aldosterone concentration (PAC: $336 \mathrm{pg} / \mathrm{mL}$ ) and low plasma renin activity (PRA: $0.4 \mathrm{ng} / \mathrm{mL} / \mathrm{h}$ ). The aldosterone/renin ratio (PAC/PRA) was also high at 840 . The patient was admitted as possible PA to the Department of Endocrinology of our hospital.

MRI with chemical shift imaging showed that the adrenal mass was lipid-rich, while a high signal intensity on diffusionweighted images suggested adenoma. The response of PRA and PAC to the furosemide upright stress test, captopril stress test, and saline infusion test showed PA. Oral administration of nifedipine $(40 \mathrm{mg} /$ day) and potassium chloride $(600 \mathrm{mg} /$ day $)$ was commenced and the patient was admitted for AVS.

To perform AVS, infusion of synthetic ACTH (tetracosactide acetate) was commenced at $0.1 \mathrm{mg} / \mathrm{h}$ and a 5 -French $(\mathrm{Fr})$ catheter was inserted via the right inguinal vein. AVS was conducted smoothly on the left side. For AVS on the right side, the standard 5-Fr RRA catheter (Terumo Co. Ltd; Tokyo, Japan) and 5-Fr right adrenal gland catheter (Medikit Co. Ltd; Tokyo, Japan) only provided poor backup, so a microcatheter (OM type 2.2-Fr Gold Crest split tip; Koshin Medical, Co. Ltd; Tokyo, Japan) was advanced into the right adrenal vein. However, the catheter was displaced by body movement with respiration

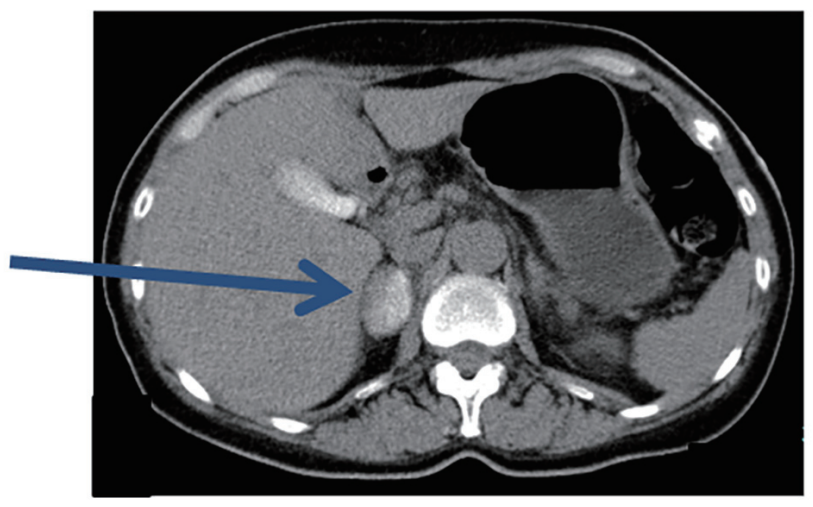

Figure 3. Plain CT after AVS. Immediately after AVS, pooling of contrast medium in the entire right adrenal gland was observed (arrow). and sampling failed. It was switched to an MK ADRENAL-R 5-Fr catheter (Koshin Medical, Co. Ltd) with stronger backup, and engagement in the right adrenal vein was achieved and imaging was performed with tip injection.

After sampling with this microcatheter, the patient complained of chest discomfort and back pain, and pooling of contrast medium was noted in the entire right adrenal gland (Fig. 2).

CT performed immediately after AVS revealed a hematoma and pooling of contrast medium in the right adrenal gland (Fig. 3). Although chest and back pain continued, the ECG and laboratory findings ruled out myocardial ischemia. Buprenorphine was administered for the pain and the patient was kept in hospital for bed rest. Her symptoms improved after 3 days and she was discharged.

The results of AVS at 15 sites are shown in Figure 4. Whether a catheter has been inserted into the adrenal vein is judged from the plasma cortisol concentration (PCC) following ACTH stimulation, which should be $\geq 200 \mu \mathrm{g} / \mathrm{dL}$ [3]. Based on this criterion, blood sampling sites (4), (7), (8), and (9) were considered to correspond to the adrenal gland. Based on a selective index (adrenal vein PCC/inferior vena cava PCC $\times 5$ ) as the criterion [10], which was $24.3 \times 5=121.5$ in this patient, sites (3), (4), (7), (8), and (9) were considered to correspond to sampling from the adrenal gland.

When aldosterone hypersecretion was defined as PAC $\geq$ $14,000 \mathrm{pg} / \mathrm{dL}$ in the adrenal vein following ACTH stimulation [11], sites (3), (4), and (8) were judged to show hypersecretion of aldosterone. Based on a right/left difference of the PAC/ PCC ratio $\geq 2.6$ [11], sites (3) and (4) showed hypersecretion of aldosterone. From the above, PA due to a right adrenal gland adenoma was diagnosed.

The right adrenal gland hematoma was followed up by CT for 60 days (Fig. 5). Although the hematoma diminished, slight enlargement of the adrenal gland persisted. It was anticipated that necrosis due to the hematoma would lead to improvement of PA, so the patient was followed by monitoring her blood pressure, PRA, and PAC (Table 1). However, the blood pressure and aldosterone/renin ratio did not decline. 


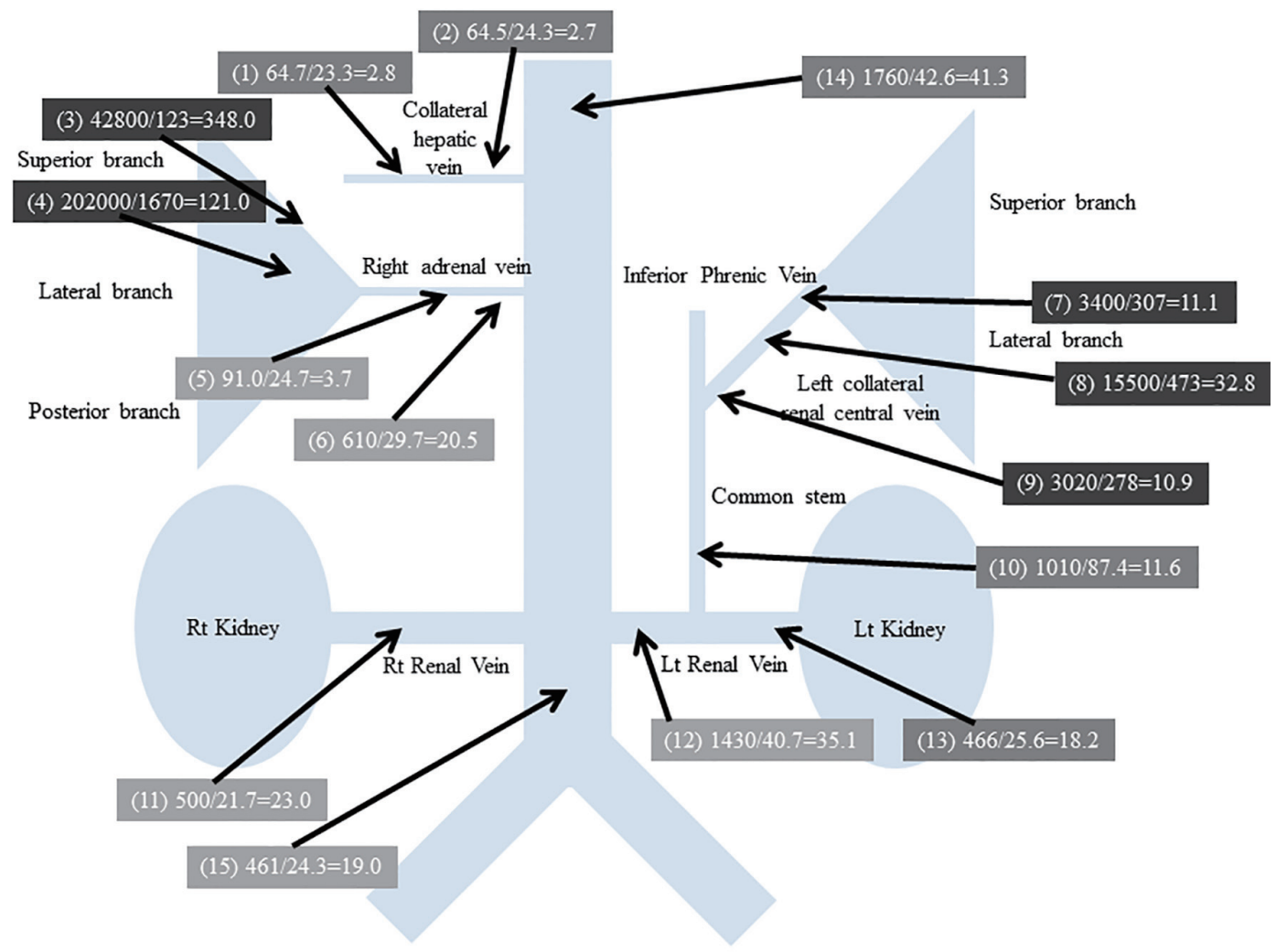

Figure 4. Results of AVS. Blood sampling site numbers are in parentheses. Plasma aldosterone concentration $(\mathrm{pg} / \mathrm{mL}) / \mathrm{plasma}$ renin activity $(\mathrm{ng} / \mathrm{mL} / \mathrm{h})=$ aldosterone-renin ratio.

At 68 days after AVS, laparoscopic right adrenalectomy was performed. The blood pressure and aldosterone/renin ratio improved after surgery, and the blood pressure was normalized without oral medication.

Pathological examination of the resected adrenal gland showed that the tumor was heterogeneous, ranging from large lipid-rich cells to smaller cells with relatively little lipid and cells with compact eosinophilic cytoplasm (Fig. 6). The histological features were consistent with a diagnosis of adrenal cortical adenoma. The adrenal gland showed focal necrosis, hemorrhage cystic change, a mild chronic inflammatory infiltrate, mild interstitial fibrosis, foreign body giant cells, and hemosiderin-laden macrophages. These changes were considered to be secondary to $\mathrm{AH}$ and the adenoma was largely spared.

\section{Discussion}

$\mathrm{AH}$ is an important complication of AVS and occurs due to adrenal vein rupture, dissection, infarction, or thrombosis [12]. $\mathrm{AH}$ is usually associated with severe pain requiring opioid analgesia. However, the hemorrhage itself does not usually need invasive treatment or blood transfusion [8].

The incidence of AH associated with AVS is reported to be $0.6 \%$ [12]. It has been suggested that limited procedural experience leads to a higher incidence of $\mathrm{AH}$ [12], but it was also
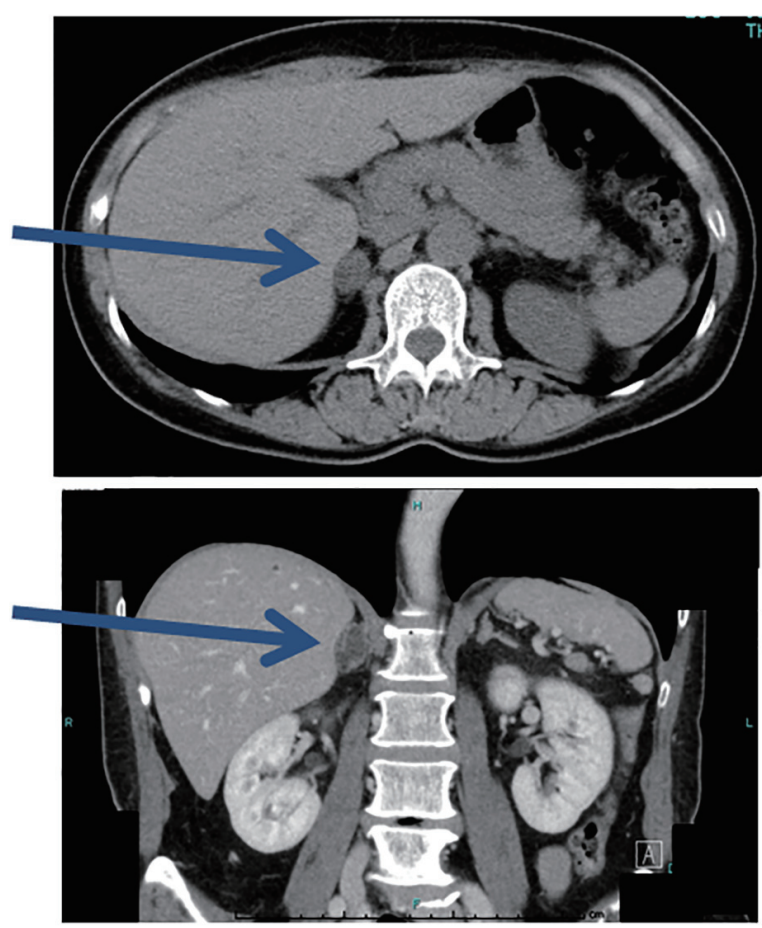

Figure 5. Plain CT at 60 days after AVS. The hematoma is smaller, but the adrenal gland is slightly enlarged (arrows). 
Table 1. Changes of BP, PR and Endocrine Markers

\begin{tabular}{llll} 
& Before AVS (nifedipine $\mathbf{4 0} \mathbf{~ m g}$ ) & 2 weeks after AVS (nifedipine $\mathbf{4 0}$ mg) & After adrenalectomy (no medication) \\
\hline $\mathrm{SBP}, \mathrm{mm} \mathrm{Hg}$ & 136 & 137 & 121 \\
$\mathrm{DBP}, \mathrm{mm} \mathrm{Hg}$ & 80 & 95 & 83 \\
$\mathrm{PR}, \mathrm{bpm}$ & 78 & 92 & 67 \\
$\mathrm{PRA}, \mathrm{ng} / \mathrm{mL} / \mathrm{h}$ & 0.2 & 0.1 & 0.4 \\
Cortisol, $\mu \mathrm{g} / \mathrm{dL}$ & 9.9 & 9.1 & 7.2 \\
PAC, $\mathrm{pg} / \mathrm{mL}$ & 355 & 105 & 124 \\
ARR & 1,775 & 1,050 & 310 \\
\hline
\end{tabular}

PR: pulse rate; PRA: plasma renin activity; PAC: plasma aldosterone concentration; ARR: aldosterone-renin ratio.

reported that occurrence of $\mathrm{AH}$ was unrelated to the experience of the operator [8]. While AH may not be closely related to procedural experience, it tends to occur more often on the right side [8]. This is probably due to the vascular anatomy since the right adrenal vein has a smaller diameter and directly enters the inferior vena cava. Accordingly, catheter engagement is more difficult on the right side [9].

The present patient had PA due to a right adrenal adenoma. Since AH affected the gland with the tumor, we expected that adrenal dysfunction due to tissue necrosis would ameliorate the patient's PA. However, her blood pressure and aldosterone/ renin ratio did not improve during follow-up. Subsequently, pathological examination of the resected specimen indicated that the adenoma had not been significantly affected by hemorrhage. According to a previous report about three patients with AH on the side of the APA, hemorrhagic damage was observed in both the tumors and adjacent adrenal tissue, but none of the patients developed adrenal insufficiency and PA did not resolve either [8]. Rather than curing PA, occurrence of AH makes laparoscopic adrenalectomy more difficult due to adhesions. Thus, bleeding from the adrenal vein may not have much effect on the tumor despite extensively involving

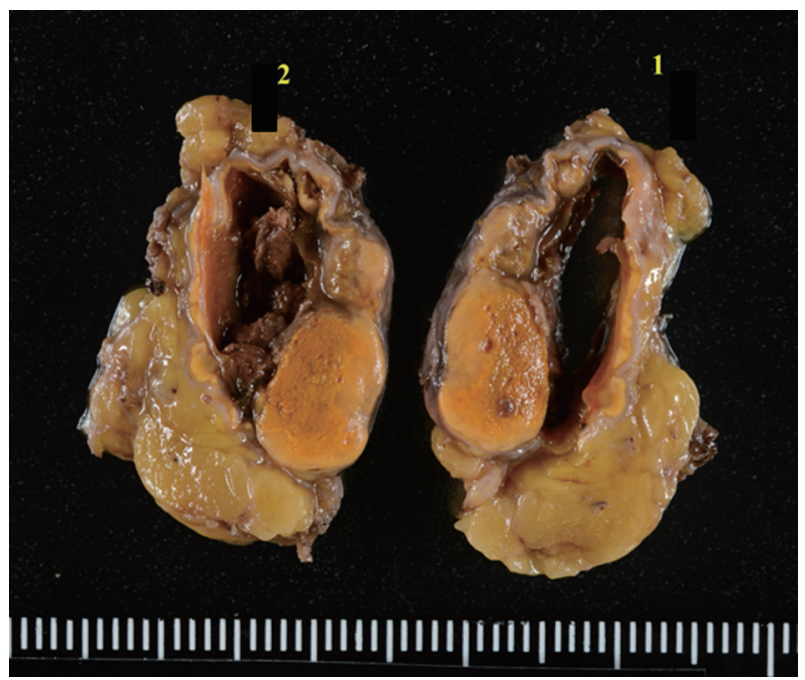

Figure 6. Cut surfaces of the resected right adrenal gland. The adrenal gland shows focal necrosis and hemorrhage cystic change, probably due to AVS. the adrenal gland.

In patients with aldosterone-producing microadenoma or idiopathic hyperaldosteronism and no obvious tumor mass, AH may reduce aldosterone production by damaging the adrenal gland. It has been reported that if $\mathrm{AH}$ occurs opposite to the APA, long-term corticosteroid replacement may be needed due to adrenal insufficiency after adrenalectomy, so careful followup should be conducted in patients with contralateral $\mathrm{AH}$ [8]. Specifically, AH frequently occurs on the right side, so right AVS should be conducted with great care in patients with APA of the left adrenal gland.

There have been few comprehensive reports about $\mathrm{AH}$ and there is no consensus regarding its management. AVS is conducted before surgical intervention and a clear result is needed. It was reported that performing CT to assess the anatomy of the right adrenal vein improves the success rate of AVS and shortens the procedure time [9]. Accordingly, performing CT is essential before this procedure and should reduce the risk of $\mathrm{AH}$.

If cannulation of the adrenal vein can be conducted easily, the incidence of $\mathrm{AH}$ would also be reduced. Rapid measurement of cortisol during AVS is effective [13], and increases the success rate of this procedure [14]. However, equipment for rapid measurement is expensive and not readily available, so clinical adoption is difficult. Furthermore, rapid measurement of cortisol during AVS requires stable engagement of the cannula for about $30 \mathrm{~min}$. A qualitative cortisol assay based on immunochromatography has been developed as a test kit, but it is not yet available commercially [15].

Cone beam CT rotational angiography with $3 \mathrm{D}$ reconstruction of retrograde venous contrast images can accurately locate the catheter [16] and we employ this method for right AVS. During contrast imaging, the medium should be injected carefully via a microcatheter to prevent $\mathrm{AH}$.

Even after successful engagement of a catheter in the adrenal vein, the tip of the catheter can easily be disengaged as the adrenal gland moves with respiration and body movements. In order to prevent this, the patient should be instructed to avoid deep breathing and should keep as still as possible. Moreover, unnecessary conversation with the patient should be avoided.

When the tip of a standard microcatheter is in close contact with the vein wall, the vein may collapse and blood sampling will become difficult. Accordingly, we used a 5-Fr cath- 
eter and a microcatheter (OM type 2.2 Fr Gold Crest $\left.{ }^{\circledR}\right)$ for AVS on the right side. The OM type microcatheter had a diameter of $0.73 \mathrm{~mm}$ and side holes near the tip that prevented complete obstruction by contact with the vein wall.

We also used an MK ADRENAL-R 5-Fr to stabilize the catheter. However, use of a back-up catheter may increase the risk of $\mathrm{AH}$. When a back-up catheter is employed, care must be taken to avoid dissection as with coronary procedures.

Moreover, when the guidewire and microcatheter are advanced together, the microcatheter occupies the inner lumen of the 5-Fr catheter and contrast injection via the catheter cannot be done. If the guidewire is removed from the microcatheter, contrast imaging or blood sampling is possible, but a 5-Fr catheter is easily disengaged when the wire is removed. If a 6-Fr catheter is used instead, a microcatheter can be advanced to the segmental adrenal vein branch with a $0.018 \mathrm{~mm}$ guidewire while contrast imaging is conducted. Accordingly, this method could improve safety. In addition, a larger catheter has stronger back-up. A 6-Fr RDC1 catheter for percutaneous transluminal renal angioplasty can achieve good engagement, but is expensive. A 6-Fr ITA1 diagnostic catheter is inexpensive and can also be used. However, 6-Fr diagnostic catheters for coronary procedures are rarely available, since 4- to 5-Fr catheters are mainly used. We previously used a 6-Fr ITA1 catheter. At present, a 6.5-Fr tapered tip sampling catheter is available for AVS. However, at the time of obtaining a catheter, we found that AVS with 6-Fr or larger catheters is rarely conducted in western Japan and such catheters are not well known. It seems that there are regional variations of the AVS technique in Japan.

After unilateral or bilateral hypersecretion of aldosterone is diagnosed by AVS, total adrenalectomy on the affected side is the standard treatment for functioning adenomas such as an APA or cortisol-producing adenoma. However, partial adrenalectomy including the tumor is performed at some institutions. For partial adrenalectomy, detailed localization of the site of excessive aldosterone secretion inside the adrenal gland is performed by segmental adrenal venous sampling (SAVS) [17-19]. If excessive hormone production is confined to the tumor itself, partial resection of the adrenal gland including the tumor is conducted while retaining the normal adrenal tissues. In patients with bilateral lesions, it has been reported that subtotal adrenalectomy is effective [20], and surgical treatment of both adrenal glands for bilateral APA is possible without postoperative adrenal failure [21]. To perform S-AVS, it is necessary to advance a $0.018 \mathrm{~mm}$ wire and a microcatheter deep into the adrenal gland. Accordingly, if $\mathrm{S}$-AVS becomes more popular, the incidence of $\mathrm{AH}$ will probably increase [18].

If $\mathrm{AH}$ leads to hemodynamic instability, emergency resection of the affected adrenal gland will be required. If surgery cannot be conducted promptly, trans-catheter adrenal arterial embolization [22], which is a treatment option for unilateral $\mathrm{PA}$, may be effective for $\mathrm{AH}$.

Because PA is a benign disease, AH should be avoided when AVS is performed for preoperative assessment. In order to conduct AVS in a safe and reliable manner, it is important to take appropriate measures to prevent $\mathrm{AH}$ before and during the procedure.

\section{Conflict of Interest}

The authors state that they have no conflict of interest.

\section{Grants}

There was no support for this case report.

\section{References}

1. Omura M, Saito J, Yamaguchi K, Kakuta Y, Nishikawa T. Prospective study on the prevalence of secondary hypertension among hypertensive patients visiting a general outpatient clinic in Japan. Hypertens Res. 2004;27(3):193202.

2. Milliez P, Girerd X, Plouin PF, Blacher J, Safar ME, Mourad JJ. Evidence for an increased rate of cardiovascular events in patients with primary aldosteronism. J Am Coll Cardiol. 2005;45(8):1243-1248.

3. Omura M, Sasano H, Saito J, Yamaguchi K, Kakuta Y, Nishikawa T. Clinical characteristics of aldosterone-producing microadenoma, macroadenoma, and idiopathic hyperaldosteronism in 93 patients with primary aldosteronism. Hypertens Res. 2006;29(11):883-889.

4. Conn JW, Louis LH. Primary aldosteronism: a new clinical entity. Trans Assoc Am Physicians. 1955;68:215-231; discussion, 231-213.

5. Omura M, Sasano H, Fujiwara T, Yamaguchi K, Nishikawa T. Unique cases of unilateral hyperaldosteronemia due to multiple adrenocortical micronodules, which can only be detected by selective adrenal venous sampling. Metabolism. 2002;51(3):350-355.

6. Doorenbos H, Elings HS, Van Buchem FS. Primary aldosteronism due to adrenocortical hyperplasia. Lancet. 1956;271(6938):335-337.

7. Kempers MJ, Lenders JW, van Outheusden L, van der Wilt GJ, Schultze Kool LJ, Hermus AR, Deinum J. Systematic review: diagnostic procedures to differentiate unilateral from bilateral adrenal abnormality in primary aldosteronism. Ann Intern Med. 2009;151(5):329-337.

8. Monticone S, Satoh F, Dietz AS, Goupil R, Lang K, Pizzolo F, Gordon RD, et al. Clinical management and outcomes of adrenal hemorrhage following adrenal vein sampling in primary aldosteronism. Hypertension. 2016;67(1):146-152.

9. Young WF, Stanson AW. What are the keys to successful adrenal venous sampling (AVS) in patients with primary aldosteronism? Clin Endocrinol (Oxf). 2009;70(1):14-17.

10. Ceral J, Solar M, Krajina A, Ballon M, Suba P, Cap J. Adrenal venous sampling in primary aldosteronism: a low dilution of adrenal venous blood is crucial for a correct interpretation of the results. Eur $\mathrm{J}$ Endocrinol. 2010;162(1):101-107.

11. Nishikawa T, Omura M, Satoh F, Shibata H, Takahashi K, Tamura N, Tanabe A, et al. Guidelines for the diagnosis and treatment of primary aldosteronism--the Japan Endo- 
crine Society 2009. Endocr J. 2011;58(9):711-721.

12. Rossi GP, Barisa M, Allolio B, Auchus RJ, Amar L, Cohen D, Degenhart C, et al. The Adrenal Vein Sampling International Study (AVIS) for identifying the major subtypes of primary aldosteronism. J Clin Endocrinol Metab. 2012;97(5):1606-1614.

13. Monticone S, Mulatero P. Rapid cortisol assay increases the success of adrenal vein sampling. Am J Hypertens. 2011;24(12):1265.

14. Rossi E, Regolisti G, Perazzoli F, Negro A, Grasselli C, Santi R, Cavalieri S, et al. Intraprocedural cortisol measurement increases adrenal vein sampling success rate in primary aldosteronism. Am J Hypertens. 2011;24(12):1280-1285.

15. Yoneda T, Karashima S, Kometani M, Usukura M, Demura M, Sanada J, Minami T, et al. Impact of new quick gold nanoparticle-based cortisol assay during adrenal vein sampling for primary aldosteronism. J Clin Endocrinol Metab. 2016;101(6):2554-2561.

16. Higashide T, Funabashi N, Tanaka T, Inoue K, Kazama T, Motoori K, Nagano H, et al. Detection of adrenal veins on selective retrograde CT adrenal venography in comparison with digital subtraction angiography in subjects with established diagnosis of one-sided adrenal aldosterone-producing tumor confirmed by adrenal vein sampling, histopathology and clinical course. Int J Cardiol. 2013;168(4):3254-3258.

17. Omura M, Saito J, Matsuzawa Y, Nishikawa T. Supper- selective ACTH-stimulated adrenal vein sampling is necessary for detecting precisely functional state of various lesions in unilateral and bilateral adrenal disorders, inducing primary aldosteronism with subclinical Cushing's syndrome. Endocr J. 2011;58(10):919-920.

18. Satani N, Ota H, Seiji K, Morimoto R, Kudo M, Iwakura $\mathrm{Y}$, Ono Y, et al. Intra-adrenal aldosterone secretion: segmental adrenal venous sampling for localization. Radiology. 2016;278(1):265-274.

19. Satoh F, Morimoto R, Seiji K, Satani N, Ota H, Iwakura Y, Ono $\mathrm{Y}$, et al. Is there a role for segmental adrenal venous sampling and adrenal sparing surgery in patients with primary aldosteronism? Eur J Endocrinol. 2015;173(4):465477.

20. Sukor N, Gordon RD, Ku YK, Jones M, Stowasser M. Role of unilateral adrenalectomy in bilateral primary aldosteronism: a 22-year single center experience. J Clin Endocrinol Metab. 2009;94(7):2437-2445.

21. Morimoto R, Satani N, Iwakura Y, Ono Y, Kudo M, Nezu $\mathrm{M}$, Omata K, et al. A case of bilateral aldosterone-producing adenomas differentiated by segmental adrenal venous sampling for bilateral adrenal sparing surgery. J Hum Hypertens. 2016;30(6):379-385.

22. Kometani M, Yoneda T, Demura M, Karashima S, Mori S, Oe M, Sawamura T, et al. The long-term effect of adrenal arterial embolization for unilateral primary aldosteronism on cardiorenovascular protection, blood pressure, and the endocrinological profile. Intern Med. 2016;55(7):769-773. 